\title{
Influencing Software Usage
}

\author{
Lorrie Faith Cranor Rebecca N. Wright \\ AT\&T Labs - Research \\ \{lorrie, rwright\}@research.att.com
}

\begin{abstract}
Technology designers often strive to design systems that are flexible enough to be used in a wide range of situations. Software engineers, in particular, are trained to seek general solutions to problems. General solutions can be used not only to address the problem at hand, but also to address a wide range of problems that the designers may not have even anticipated. Sometimes designers wish to provide general solutions, while encouraging certain uses of their technology and discouraging or precluding others. They may attempt to influence the use of technology by "hard-wiring" it so that it only can be used in certain ways, licensing it so that those who use it are legally obligated to use it in certain ways, issuing guidelines for how it should be used, or providing resources that make it easier to use the technology as the designers intended than to use it in any other way.

This paper examines several cases where designers have attempted to influence the use of technology through one of these mechanisms. Such cases include key recovery encryption, Pegasus Mail, Platform for Internet Content Selection (PICS) Guidelines, Java, Platform for Privacy Preferences Project (P3P) Implementation Guide, Apple's style guidelines, and Microsoft Foundation Classes. In some of these cases, the designers sought to influence the use of technology for competitive reasons or in order to promote standardization or interoperability. However, in other cases designers were motivated by policy-related goals such as protecting privacy or free speech. As new technologies are introduced with the express purpose of advancing policy-related goals (for example, PICS and P3P), it is especially important to understand the roles designers might play in influencing the use of technology.
\end{abstract}

\section{Introduction}

Technology designers often strive to design systems that are flexible enough to be used in a wide range of situations. Software engineers, in particular, are trained to seek general solutions to problems. General solutions can be used not only to address the problem at hand, but also to address a wide range of problems that the designers may not have even 
anticipated. For example, spreadsheet programs often come with templates for computing interest payments, keeping track of personal finances, and performing other operations that their designers consider useful. But users can also use these programs to record and analyze virtually any kind of tabular data. Thus these programs are not limited by the ability of their designers to anticipate ways that spreadsheets will be used. Indeed, users can and do invent their own spreadsheet applications.

While general technology solutions allow users to use the technology as they see fit, users may not always use the technology in desirable ways. Users may use general-purpose technology tools in ways that are inefficient, dangerous, or contrary to the spirit in which the technology was designed.

The Hypertext Transfer Protocol (HTTP) Referer field provides an example of the danger of using technology in an unintended way. HTTP is the protocol used for requesting and transferring Web pages over the Internet BLFF96. The Referer field is an optional HTTP header that allows Web site operators to track the addresses of Web pages from which visitors to their site are referred. However, some Web sites use the Referer field for another purpose for which it was never intended: to verify that forms submitted by visitors have not been improperly modified. This is a very weak authentication mechanism that can be defeated easily by forging the Referer field's contents. Furthermore, as the Referer field is an optional header, there is no guarantee that Web browsers will transmit it to a Web site. Indeed, to address a security problem, Microsoft modified the behavior of the Internet Explorer browser beginning with version 4.0 so that the Referer field is not transmitted under certain conditions Mic98. As a result, some sites that use the Referer field in this unintended way are finding that users are unable to submit forms at their site. In a column describing this problem, Lincoln D. Stein concluded, "This painful lesson illustrates why it's almost never a good idea to use a feature for other than its intended purpose" [Ste98.

Some unintended usage of technology is neither inefficient nor dangerous, but it nonetheless violates the spirit in which the technology was designed. For example, some email programs are efficient tools for sending out bulk email messages. One such tool, Pegasus Mail, was developed by programmer David Harris to provide a free service "that enhances the quality of communication between people" Har98b. However, many people have used 
Pegasus mail to send unsolicited commercial email, better known as "spam." Spam is often blamed for interfering with interpersonal communication, increasing the time and effort necessary to retrieve and identify desired email CL98. Thus when Pegasus Mail is used for sending spam it violates the spirit in which this technology was designed.

Sometimes designers wish to provide general solutions, while encouraging certain uses of their technology and discouraging or precluding others. In this paper we identify four types of mechanisms used by software engineers (or their employers) to influence the use of technology. Software engineers may attempt to influence the use of technology by:

- "hard-wiring" it so that it only can be used in certain ways,

- licensing it so that those who use it are legally obligated to use it in certain ways,

- issuing guidelines for how it should be used, or

- providing resources that make it easier to use the technology as the designers intended than to use it in any other way.

We provide several examples of software systems to which each of these mechanisms has been applied. In some of these cases, the designers sought to influence the use of technology for competitive reasons or in order to promote standardization or interoperability. However, in other cases designers were motivated by policy-related goals such as protecting privacy or free speech. In some cases, the designers did not actually seek to influence the use of technology, but their actions had inadvertent influence.

As new technologies are introduced with the express purpose of advancing policy-related goals (for example, PICS and P3P), it is especially important to understand the roles designers might play in influencing the use of technology.

\section{Hard-Wiring}

By "hard-wiring" software systems, designers can ensure that software behaves in a certain way and thus influence how it can be used. 


\subsection{Key Recovery Encryption}

Key recovery encryption, also sometimes called key escrow encryption or trusted third-party encryption, is the term for encryption schemes in which the secret encryption keys may be recovered by certain parties in certain circumstances.

The U.S. government advocates key recovery encryption as providing a balance between the need on the one hand for individual privacy and legitimate commercial activities, and on the other hand the need for government eavesdropping for purposes of law enforcement or national security MK97. However, not everyone agrees that these concerns necessarily conflict, nor that such a balance is best provided by key recovery encryption $\mathrm{AAB}^{+} 97$.

The U.S. government claims any key recovery encryption system will be voluntary to use [Adm96], and that citizens will be free to use whatever encryption software they wish (though some also fear that this is merely a stepping stone to disallowing all non-recoverable encryption systems, as has already been done in countries such as France, Russia, and Indonesia). However, the government hopes that key recovery encryption will become widely used by hard-wiring it into standardized, widely available solutions, such as secure telephones, Web browsers, and a global public key infrastructure [Dep96]. Furthermore, the government can further propagate the use of its sanctioned key recovery encryption solutions by allowing them to be exported freely to other countries, while strong encryption solutions that do not allow key recovery are currently heavily restricted against export [Dep96].

Indeed, despite industry's initial opposition to key recovery encryption, several companies have realized that because of the government's willingness to provide export licenses for key recovery encryption and not to routinely provide them for products including nonrecoverable encryption, it may be commercially more viable to support key recovery. To this end, over 30 companies from around the world have formed the "Key Recovery Alliance" (KRA) to "promote the implementation, deployment, and use of interoperable key recovery technologies in the global marketplace." [Key97]. KRA members include Apple, IBM, Digital Equipment Corporation, Hewlett-Packard, and Verisign. In addition, other companies, such as Netscape, have agreed to deploy key recovery encryption in order to obtain export licenses for their products. As more businesses routinely include key recovery encryption in their 
products, more users will use key recovery encryption even if they are unaware that this is the case; this is discussed more in Section 5.1.

\subsection{Pegasus Mail}

In order to prevent users of Pegasus Mail from using it to send spam, Harris might have hardwired Pegasus Mail so that it could not be used in this way. However, this would have likely reduced the program's utility as a communication tool, making it difficult or impossible for people to use it to send legitimate bulk email. Harris took a different approach and modified Pegasus Mail (beginning with version 2.50) so that it automatically inserts "X-Distribution" headers into all email messages it sends that are distributed to 50 or more recipients. Harris also distributed instructions for filtering spam identified by the presence of these headers. Harris explained that this step should reduce "the abuse of Pegasus Mail ... yet it does not significantly impact on legitimate users of the program" [Har97. As discussed in 3.1, Harris also uses licensing to control the use of Pegasus Mail for sending spam.

\subsection{PICS Implementations}

The Platform for Internet Content Selection (PICS) is a series of specifications developed by the World Wide Web Consortium (W3C) to provide a standard platform for rating Internet content. PICS allows anyone to come up with a set of rating criteria - called a rating system - and use it to rate Web pages. The development of PICS was largely motivated by a desire to give parents tools they could use to protect their children from harmful online materials (as an alternative to laws that would prohibit the online distribution of materials harmful to children). However, PICS was designed to be a more general specification for content rating that would be useful for a wide variety of applications beyond protecting children [RM96. But despite the flexibility of the PICS specifications, most PICS implementations to date have hard-wired settings that make them ill-suited for many applications that go beyond protecting children. The Netscape Navigator 4.5 implementation even goes so far as to hard-wire in two specific PICS rating systems.

Content Advisor - the PICS implementation in the Microsoft Internet Explorer Web 
browser - has a user interface that will read content labels from any PICS rating system, but can only be configured to select content with numeric ratings less than a specified threshold. This makes sense for some of the most common PICS ratings systems that rate characteristics such as 'nudity' and 'violence' on increasing numeric scales. A parent who wants to prevent her child from viewing content with a violence rating higher than 3 simply positions the slider on the Content Advisor interface so that only content with violence rating 3 or lower is permitted. The interface is hard-wired so that it is impossible to configure it to accept only content rated as having high levels of nudity or violence (or any other criteria) or to select ratings in the middle of a range Cra98a. It is not clear whether the interface designers restricted the interface so as to thwart efforts by children to seek out sexual or violent content, or whether the interface was designed this way for other reasons (or even arbitrary reasons). However, it has the effect of limiting the ways that Content Advisor can be used. A likely unintended consequence is that the interface cannot be used to filter out low quality content using a PICS rating system that assigns numeric ratings that correspond to quality stars (four stars for high quality content, zero stars for low quality content, etc.).

\subsection{Default Settings}

Even when they are not "hard-wired" and may even be relatively easy to change, the settings initially provided by the manufactures are often left untouched by users. Consequently, the default settings that inevitably accompany most pieces of software often have great influence on how that software is used. In some cases, designers are cognizant of this and design the default settings with an eye towards influencing user behavior. In other cases, default settings are based on somewhat arbitrary decisions, but they may be nonetheless influential. There is evidence that users rarely change default settings in popular consumer software products. For example, Netscape reports that 40 percent of the visitors to their Web site come there because they have not changed their default home page [Sei98].

When designing software that includes features that can be configured in multiple ways, designers are often faced with a choice of (a) configuring the features with default settings,

(b) leaving the features that require configuration turned off, or (c) leaving features that 
require configuration unconfigured, but prompting users to configure them before they can use the software [CR97. While explicitly configuring features with default settings is an

obvious way to influence software use, leaving features that require configuration turned off can also be influential as it limits the degree to which those features will likely be used at all.

\section{Licensing}

Software may be licensed so that those who use it are legally obligated to use it in certain ways.

\subsection{Pegasus Mail}

In addition to hard-wiring Pegasus Mail to insert special headers when the program is used to send bulk email as described in Section 2.2, Harris also wrote a prohibition against spamming into the Pegasus Mail license. The Pegasus Mail Terms and Conditions of Use specifically prohibits using Pegasus Mail for sending email to "more than 50 recipients for the purpose of advertising a commercial product or service, where the recipient has not explicitly expressed interest in receiving such advertisements." The license also prohibits including the program in a package designed for sending spam or promoting the use of the program for that purpose [Har98b]. However, Harris speculates that the cost of enforcing this license in the U.S. legal system would likely be prohibitive for him Har98a. Indeed while the threat that licenses may be enforced may be somewhat effective in deterring unauthorized use, the ultimate effectiveness of licenses is likely to depend on their ability to be enforced.

\section{$3.2 \quad$ Java}

Sun Microsystems' Java technology is designed to allow software developers to create "writeonce, run-anywhere" code. That is, any code written in Java should run on any computer, or in any Web browser, that supports Java, without the developer having to know anything about which computers or browsers the code will be run on. The Java technology license 
carries two major restrictions on its use. The first places restrictions on its use for critical applications that it may not be suitable for, while the second ensures compatibility among different Java language based products, thereby maintaining the write-once, run-anywhere philosophy.

More specifically, the first restriction, found in the Java Developer's Kit (JDK) on-line binary code license agreement applies to anyone developing Java applications, and states that the "software is not designed or licensed for use in on-line control equipment in hazardous environments such as operation of nuclear facilities, aircraft navigation or control, or direct life support machines" [Sun97]. While this may be hard to enforce due to the problem of detection before problems occur, it makes clear Sun's impunity to lawsuits in the event that such use of Java results in damages.

The second restriction is only required for a commercial source license, needed if the user intends to merge the Java source code into a commercial product, such as a commercial Web browser that supports the Java interpreter and class libraries. The commercial source code license is devoted to ensuring cross-platform Java compatibility. Compatibility requirements are ensured partly by restrictions on the kinds of changes that can be made to the underlying Java technology components provided by Sun, and are tested through a detailed series of "test suites." For example, the commercial source license between Sun and Microsoft includes the following [SM96]:

Licensee agrees that upon ... (a) six (6) months after the date that SUN delivers to Licensee an Upgrade that SUN designates a significant Upgrade (each, a "Significant Upgrade"), ... Licensee shall deliver to SUN ... an upgrade to the Java Reference Implementation (each, a "Compatible Implementation") that passes the test suite that accompanied the Significant upgrade (a "Relevant Test Suite")...

Licensee shall confine the names of all VAOPs [Value Added Open Packages] to names beginning with "COM.ms" and shall not modify or extend the names of public class or interface declarations whose names begin with "java", "COM.sun" or their equivalents... 
Licensee agrees that the Reference Implementation VM [Virtual Machine], and any upgrades thereto, shall include the necessary Source Code to implement the functionality of the Java Runtime Interpreter. Notwithstanding the foregoing, Licensee may implement a subset of the functionality. . callable by a system-level interface (a "System Implementation"). In such cases, Licensee shall provide SUN a complete and accurate specification for all such system-level interfaces...

Licensee agrees that the Reference Implementation Java Classes shall be maintained as an application level library.

The license also says that any Java classes added by the licensee to the existing set of Java classes should be made promptly available to developers and customers free of charge, and that for any "Portable Value Added Open Packages," either the source code or a complete and accurate specification including an appropriate test suite must be made publicly available.

Because Sun views cross-platform compatibility as a fundamentally important aspect of Java, it has been aggressive about enforcing such requirements. Indeed, Sun sued Microsoft in October of 1997 for alleged breaches of their license in Microsoft's Internet Explorer 4.0 resulting in incompatibilities with the Java standard. At the time of this writing, the suit still continues, with hearings scheduled in September 1998.

\subsection{PICS}

Licensing has been considered and rejected for some technologies due in part to the difficulty of defining the scope of a license and following up with enforcement. For example, PICS was intended to be an alternative to legislative proposals that would ban or restrict certain kinds of content; PICS was designed to be a voluntary system that would let parents decide for themselves what content is appropriate for their children [RM96]. However, PICS drew criticism from free speech advocates who were concerned that one or two PICS rating systems could become de facto standards and eventually become subject to legislation requiring their use [Ame97]. In response to frequent questions about why the PICS designers had not licensed PICS so as to preclude its being used in ways that might be harmful to free speech, one of the PICS designers responded that at the time PICS was developed licensing was 
viewed as infeasible because it would "undercut the "neutrality' and appeal of the technology" and because "the W3C then would have had to be in the position of determining who should and should not use it; this is not a role the W3C is competent to play" Res98.

\section{Guidelines}

Designers sometimes issue guidelines about how software or systems should be used. Guidelines do not limit the ability of technology to be used in unexpected ways, but they can provide guidance as to the way technology should be used. Guidelines can also be issued after software is released and they can be updated as people gain experience using a technology.

While guidelines can make explicit the intention of designers and provide guidance to those who care to take advantage of them, they are not requirements, and they often can be overlooked or ignored. The impact of guidelines may be increased by logo or seal programs that provide a logo or seal of approval that products following a particular set of guidelines are allowed to display. However, these programs generally require an arbiter to determine which products are entitled to display the logo, and permission to display the logo may be tied to a licensing agreement.

There may also be a risk that users (as well as implementors) may confuse guidelines with requirements, and come to expect that products follow published guidelines. Thus users may develop false expectations about products, or implementors may build interoperable products that interoperate only with products following a particular set of guidelines. On the other hand, if users or some implementors treat guidelines as requirements, it may put more pressure on other implementors to follow the guidelines.

\subsection{PICS}

Over two years after PICS was developed, the PICS designers issued a "Statement on the Intent and Use of PICS: Using PICS Well." This document includes a set of guidelines that address how the designers intended PICS to be used [RW98]. It's not clear that PICS had 
actually been used in an undesirable way, but criticism that PICS had been designed such that it could be used in undesirable ways (especially from a first amendment standpoint) likely prompted the PICS designers to issue this statement Ame97, Cra98a.

\section{$4.2 \mathrm{P} 3 \mathrm{P}$}

Another W3C specification, the Platform for Privacy Preferences Project (P3P) RC98, developed guidelines in parallel with the specification itself. Motivated by both the response to PICS and a perceived need to provide guidance on issues outside the scope of the P3P specification (such as user interface), the P3P designers began developing an implementation guide. One section of the guide, the P3P Guiding Principles Cra98b, provides a set of guidelines for the way P3P should be implemented and used. These guidelines emphasize that P3P was designed as a privacy protection tool and advise implementors to make design decisions that support the goals of P3P. However, the document notes that the guidelines are recommendations rather than requirements.

\subsection{Apple Style Guidelines}

Apple Computers has a deeply thought-out philosophy behind the design of its computers and their software, reflected in almost every detail, from the use of color to the placement of menus. In order to help ensure a "look and feel" consistent with this philosophy across all software applications that run on the Macintosh, Apple Computers has provided an extensive list of "style guidelines" to be used by developers of software applications for the Macintosh. Specifically, their 400-page book Macintosh Human Interface Guidelines App92b "describes the way to create products that optimize the interactions between people and Macintosh computers. It explains the whys and hows of the Macintosh interface in general terms and specific details." App92b]. This book is comprehensive and easy-to-read, with lots of examples of "good" and "bad" software development for the Macintosh; it is available both for free in electronic format from the Apple Web site, and in print from conventional booksellers. In addition, other style guidelines documents such as "Control Layout Guidelines," "Guidelines for Content Manipulation," and "Application Layout Guidelines" are available 
electronically from their Web site. In making such information available and readable, Apple has made it much easier for developers to consistently design products that fit the Macintosh model and philosophy, and many developers have indeed done so. The guidelines are also supported by a toolkit designed to help developers follow the guidelines; this is discussed below in Section 5.2.

\section{$5 \quad$ Toolkits and other Resources}

Often, the designers of software and computer systems provide resources that make it easier to use the technology as the designers intended than to use it in any other way. Such resources can be in the form of toolkits, published interface specifications, or even simply by making certain off-the-shelf solutions widely available.

\subsection{Key Recovery Encryption}

As an example of influencing usage via off-the-shelf availability, the U.S. government may succeed in its goal of making most encryption that people use recoverable simply by getting most commercial software developers to include it hard-wired in their systems as discussed in Section 2.1. Similarly, if a global public key infrastructure using key recovery is put into place, the comparative ease of using it rather than having to do encryption without the benefit of a public key infrastructure may go a long way towards making most encrypted traffic recoverable.

On the other hand, people who don't like key recovery can try to combat this by making widely available non-recoverable encryption solutions. For example, until its recent acquisition by Network Associates, the PGP (Pretty Good Privacy) encryption system Zim95 specifically did not allow key recovery, was freely available on the Internet for personal, noncommercial use, and built up a "grass-roots" public key infrastructure in which individual users certify each other's public keys. PGP's creator, Phil Zimmerman, also used PGP as a staging ground for debates on individuals' rights to privacy, cryptography export regulations, and certain patentability issues. 


\subsection{Apple Style Guidelines}

As described in Section 4.3, Apple has extensive guidelines describing what it considers to be good software development practices for the Macintosh. To further encourage developers to follow their guidelines, they also provide a toolkit of Macintosh system software routines that follow the guidelines and can be used by developers in their Macintosh software applications App92a. Since it is often easier to use the toolkit routines than to write similar routines from scratch, this results in different developers using the same toolkit routines, thereby creating a more consistent and standard interface to be presented to the user even when using software from different developers.

\subsection{Microsoft Foundation Classes}

The Microsoft Foundation Class Library (MFC) is a software library consisting of a "set of reusable, pre-built $\mathrm{C}^{++}$components that provide a completely portable interface for applications for the Microsoft Windows, Windows 95, and Windows NT operating systems," as well as some Macintosh and Unix platforms Mic95. Its main goal is to allow developers to write applications for Windows much faster than writing them from scratch. This is an important marketing feature for Microsoft because it results in more Windows applications being available even as new versions of Windows are constantly evolving. Although the MFC to some extent results in a similar look and feel across different applications, this does not appear to have been as important a consideration for Microsoft as it was for Apple (see Sections 4.3 and 5.2). Instead, they have chosen to make the MFC quite general to allow developers to have more flexibility even though this tends to result in a more diverse look and feel across different applications.

In addition to the MFC itself, Microsoft has also provided a set of guidelines for developers who want to extend the MFC in a way that will interact well with existing MFC components Mic97. 


\section{Conclusions}

We have discussed four different types of methods that software engineers may use to attempt to influence the way their technology is used. We have provided a variety of examples of how these techniques have been used, with varying degrees of success. From our analysis, there is no one method that emerges as most successful.

- Hard-wiring certainly is effective in limiting the use of a particular tool, but it may prove overly limiting, precluding many desirable (and possibly unforeseeable) uses of that tool. Furthermore, there may be alternative tools, possibly from competing vendors, readily available to those who are determined to use the technology in ways that designers are attempting to influence against.

- Licensing can be effective when licenses are readily enforceable. However, enforcing licenses can be expensive and time-consuming, both due to the difficulty of monitoring licensees to determine when licenses are not being enforced, and due to the legal expenses involved in pursuing a case against a misbehaving licensee. While the threat of enforcement may be somewhat of a deterrent, undesirable use is not actually precluded, although it may sometimes be punished after it occurs. Licensing can also be useful to limit the liability that a software developer has if their technology is used in unapproved ways; this may exert further influence on users to follow approved usage.

- Guidelines have the advantages that they do not limit unexpected uses, and they can be updated as people gain experience with a new technology. However, they neither preclude nor punish undesirable use. Logo programs and pressure from users or implementors may encourage voluntary compliance.

- Toolkits and other resources can make it much easier for people to use technology as intended than to use it in other ways that will require them to build the necessary resources themselves. However, when people feel strongly about using technology in ways not supported by the toolkit or other available resources, they can and will develop their own resources that result in uses that designers are trying to influence against. 
Designers who wish to influence software usage would be wise to consider the tradeoffs of each of these methods when determining what approach to take in a particular situation. However, designers should also keep in mind that while these methods can be useful in persuading users who are choosing between multiple alternatives they consider acceptable, these methods are unlikely to persuade users to choose alternatives to which they are opposed.

\section{References}

[AAB ${ }^{+97]}$ Hal Abelson, Ross Anderson, Steven M. Bellovin, Josh Benaloh, Matt Blaze, Whitfield Diffie, John Gilmore, Peter G. Neumann, Ronald L. Rivest, Jeffrey I. Schiller, and Bruce Schneier. The risks of key recovery, key escrow, and trusted third-party encryption, 1997. Revised 1998. Both versions available at http://www.crypto.com/key_study.

[Adm96] Administration statement on commercial encryption policy, July 1996. Available at http://csrc.ncsl.nist.gov/keyrecovery/admin.txt.

[Ame97] American Civil Liberties Union. Fahrenheit 451.2: Is Cyberspace burning? How rating and blocking proposals may torch free speech on the internet, 1997. http://www.aclu.org/issues/cyber/burning.html.

[App92a] Apple Computer, Inc. Inside Macintosh: Macintosh Toolbox Essentials. AddisonWesley, 1992.

[App92b] Apple Computer, Inc. Macintosh Human Interface Guidelines. Addison-Wesley, 1992.

[BLFF96] T. Berners-Lee, R. Fielding, and H. Frystyk. RFC 1945 - hypertext transfer protocol - HTTP/1.0, May 1996. http://info.internet.isi.edu/innotes/rfc/files/rfc1945.txt.

[CL98] Lorrie Faith Cranor and Brian A. LaMacchia. Spam! Communications of the ACM, 41(8):74-83, August 1998. 
[CR97] Lorrie Faith Cranor and Joseph Reagle. Designing a social protocol: Lessons learned from the platform for privacy preferences. In Proceedings of the Telecommunications Policy Research Conference, Alexandria, VA, September 27-29 1997. http://www.research.att.com/ ${ }^{\circ}$ lorrie/pubs/dsp/.

[Cra98a] Lorrie Faith Cranor. Bias and responsibility in 'neutral' social protocols. Computers \& Society, pages 17-19, September 1998. http://www.research.att.com/ ${ }^{\circ}$ lorrie/pubs/dimacs-values.html.

[Cra98b] Lorrie Faith Cranor. P3P guiding prinicples. W3C NOTE-P3P10-principles19980721, July 21 1998. http://www.w3.org/TR/1998/NOTE-P3P10-principles.

[Dep96] Department of Commerce, Bureau of Export Administration. Rules and regulations. Federal Register, 61(251):68572-68587, December 1996.

[Har97] David Harris. Anti-spam measures incorporated into v2.5x, 2 April 1997. Message posted to bit.listserv.pmail.

[Har98a] David Harris, 7 August 1998. Personal email to author.

[Har98b] David Harris. Pegasus mail - terms and conditions of use, 1998. http://www.pmail.com/license.

[Key97] Key Recovery Alliance. FAQs about the Key Recovery Alliance, 1997. http://www.kra.com/FAQS1209.html.

[Mic95] Microsoft Corporation. Microsoft Foundation Class Library 4.0: $\mathrm{C}^{++}$application framework for Microsoft Windows, 1995. http://premium.microsoft.com/msdn/library/background/html/msdn_mfc4tech.htm.

[Mic97] Microsoft Corporation. Microsoft Foundation Class Library development guidelines, 1997. http://premium.microsoft.com/msdn/library/background/html/msdn_mfcgde.htm. 
[Mic98] Microsoft Technical Support. INFO: IE does not send referer header in unsecured situations. Microsoft Knowledge Base Q178066, January 191998. http://support.microsoft.com/support/kb/articles/q178/0/66.asp.

[MK97] Senator John McCain and Senator Bob Kerrey. S.909: The secure public networks act, 1997.

[RC98] Joseph Reagle and Lorrie Faith Cranor. The platform for privacy preferences. W3C NOTE-P3P-CACM-19980731, July 1998. http://www.w3.org/TR/1998/NOTEP3P-CACM/.

[Res98] Paul Resnick. PICS, censorship, \& intellectual freedom FAQ, version 1.14, January 1998. http://www.w3.org/PICS/PICS-FAQ-980126.html.

[RM96] Paul Resnick and James Miller. PICS: Internet access controls without censorship. Communications of the ACM, 39(10):87-93, 1996. http://www.w3.org/PICS/iacwcv2.htm.

[RW98] Joseph Reagle and Daniel J. Weitzner. Statement on the intent and use of PICS: Using PICS well. W3C NOTE-PICS-Statement-19980601, June 1998. http://www.w3.org/TR/NOTE-PICS-Statement.

[Sei98] Robert Seidman. Netscape now or Netscape NO? Seidman's Online Insider, March 22 1998. http://www.onlineinsider.com/html/march_22__1998.html.

[SM96] Sun Microsystems and Microsoft Corporation. Technology license and distribution agreement, 1996. http://java.sun.com/aboutJava/info/document.html.

[Ste98] Lincoln D. Stein. Referer refresher. Web Techniques, pages 10-13, September 1998.

[Sun97] Sun Microsystems. Solaris JDK: On-line binary code license agreement, 1997. http://www.sun.com/solaris/jdk/download.1.1.6/gen-legal.cgi.

[Zim95] Phil Zimmerman. The Official PGP User's Guide. MIT Press, 1995. 